\title{
Biospecimen Condition
}

National Cancer Institute

\section{Source}

National Cancer Institute. Biospecimen Condition. NCI Thesaurus. Code C70714.

A characteristic that refers to the physical and org anoleptic state of a biospecimen at time of its receipt at the laboratory. 